\title{
An Efficient Manipulation Package for Biconditional Binary Decision Diagrams
}

\author{
Luca Amarú, Pierre-Emmanuel Gaillardon, Giovanni De Micheli \\ Integrated Systems Laboratory (LSI), EPFL, Switzerland
}

\begin{abstract}
Biconditional Binary Decision Diagrams (BBDDs) are a novel class of binary decision diagrams where the branching condition, and its associated logic expansion, is biconditional on two variables. Reduced and ordered BBDDs are remarkably compact and unique for a given Boolean function. In order to exploit BBDDs in Electronic Design Automation (EDA) applications, efficient manipulation algorithms must be developed and integrated in a software package. In this paper, we present the theory for efficient BBDD manipulation and its practical software implementation. The key features of the proposed approach are (i) strong canonical form pre-conditioning of stored BBDD nodes, (ii) recursive formulation of Boolean operations in terms of biconditional expansions, (iii) performance-oriented memory management and (iv) dedicated BBDD re-ordering techniques. Experimental results show that the developed BBDD package achieves an average node count reduction of $19.48 \%$ and a speed-up factor of $1.63 \mathrm{x}$ with respect to a state-of-art decision diagram manipulation package. Employed in the synthesis of datapath circuits, the BBDD manipulation package is capable to advantageously restructure arithmetic operations producing $\mathbf{1 1 . 0 2 \%}$ smaller and $32.29 \%$ faster circuits as compared to a commercial synthesis flow.
\end{abstract}

\section{INTRODUCTION}

Efficient Boolean function representation and manipulation is essential in Electronic Design Automation (EDA). Binary Decision Diagrams (BDDs) [1]-[3] are a well established data structure for this purpose. BDDs are employed in many EDA applications, such as digital circuits synthesis [4], verification [5], testing [6], simulation [7], and others. Original BDDs are driven by the Shannon's expansion to decompose a Boolean function until the constant logic values are encountered. Reduced and ordered BDDs [3] are unique for a given variable order, i.e., canonical, thus enabling efficient logic manipulation [8], [9]. Unfortunately, there exist Boolean functions for which BDDs are not compact or even too large to be handled [10]. In order to improve the compactness of BDDs, and therefore the efficiency of their applications, canonical extensions of BDDs are proposed in literature, e.g., [11]-[14]. We refer the reader to [15] for a more complete list of BDD extensions.

In this work, we focus on Biconditional Binary Decision Diagrams (BBDDs), a promising class of canonical binary decision diagrams recently introduced in [14]. While original BDDs are based on the single-variable Shannon's expansion, BBDDs employ a two-variable biconditional expansion [14], making the branching condition at each decision node dependent on two variables per time. Such feature improves the expressive power of the binary decision diagram. Moreover, BBDDs represent also the natural and native design abstraction for emerging technologies where the circuit primitive is a comparator, rather than a switch [16]-[18]. The work in [14] first introduces the concept of BBDDs but does not provide a detailed discussion about their automated manipulation.

In this paper, we present the theory for efficient BBDD manipulation and its practical software implementation. We do not address here theoretical issues related to BBDDs, such as asymptotic size bounds or other properties, but we focus on the design of a new BBDD package to support EDA applications. The main attributes enabling efficient BBDD manipulation are (i) strong canonical form pre-conditioning of stored BBDD nodes, (ii) recursive formulation of Boolean operations in terms of biconditional expansions, (iii) performance-oriented memory management and (iv) dedicated BBDD re-ordering techniques. The developed BBDD package is open-source and available online [19]. Experimental results over the MCNC benchmark suite show that the BBDD package is $1.63 \mathrm{x}$ faster than a state-of-art decision diagram package [20] and produces $19.48 \%$ smaller logic representations. In order to showcase the interest of a BBDD package for EDA, we also propose a new synthesis approach for datapath circuits. Standard synthesis techniques face challenges to satisfactory handle datapaths, that are intensive in arithmetic operations, such as comparators and voters. Instead, BBDDs are remarkably compact for arithmetic operations as the comparator function is inherently embedded in a BBDD node functionality [14]. Motivated by this consideration, we employ the BBDD package as frontend to a commercial synthesis tool to structure arithmetic operations in datapaths. Synthesis results show that datapaths pre-structured by the BBDD package are $11.02 \%$ smaller and $32.29 \%$ faster than their counterparts directly designed by a commercial synthesis flow.

The remainder of this paper is organized as follows. Section II provides a background on traditional binary decision diagrams and existing software packages. In Section III, BBDDs are introduced with their basic definitions for reference. Section IV presents the BBDD manipulation theory and discusses the software package results. Section V presents the application of the BBDD package as front-end for datapath design and discusses the synthesis results. The paper is concluded in Section VI.

\section{BACKGROUND AND MOTIVATION}

This section provides a background on traditional binary decision diagrams and related logic manipulation packages.

\section{A. Binary Decision Diagrams}

Binary Decision Diagrams (BDDs) are data structures representing Boolean functions. The general concept of BDDs is first introduced by Lee [1] and Akers [2], but later popularized by Bryant in [3], where it is shown that, under ordering and reduction rules, BDDs are a canonical representation form. 
We refer the reader to [15] for a review about terminology and fundamentals of BDDs. The common adoption of BDDs in EDA [4]-[7] is driven by efficient BDD manipulation algorithms [8], [9] and related software implementations [20]. A BDD manipulation package can be used as a generalpurpose software for manipulating Boolean functions, task at the core of EDA. We discuss hereafter the main features of a state-of-art BDD package.

\section{B. State-of-art BDD Package Implementation}

Contemporary BDD packages, such as CUDD [20], are designed to be memory-efficient and to have fast runtime. Memory efficiency is achieved by the use of hash-tables and caches. A global hash-table, commonly called the unique table, stores the nodes of the BDDs in a strong canonical form, which is a form of data pre-conditioning to reduce the complexity of equivalence test [8]. A global cache, commonly called the computed table, is used to temporarily store performed Boolean operations in case of later use. Fast BDD manipulation is achieved by a recursive formulation of Boolean operations between existing decision diagrams [8]. Additionally, variable re-ordering algorithms [9] are commonly used to minimize the number of stored nodes and maximize the package performance.

Many BDD extensions improve the expressive power of binary decision diagrams. However, only a limited number of them are supported by an efficient manipulation theory and even fewer of them by a practical software implementation.

In this work, we present the manipulation theory and efficient software implementation for a promising class of decision diagrams [14], based on a novel equality/inequality switching paradigm.

\section{BICONDITIONAL BDD OVERVIEW}

In this section, we review Biconditional Binary Decision Diagrams (BBDDs) [14], a canonical class of binary decision diagrams where the branching condition, and its associated logic expansion, acts as a two-variable comparator function.

\section{A. BBDD Fundamentals}

A BBDD is a Direct Acyclic Graph (DAG) representing a Boolean function $f(v, w, . ., z)$. A BBDD is uniquely identified by its root, the set of internal nodes, the set of edges and the $1 / 0$-sink nodes. Each internal node (Fig. 1) in a BBDD

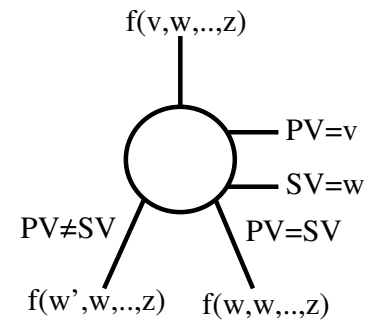

Fig. 1: BBDD internal node.

is labeled by two Boolean variables: $v$, the Primary Variable $(P V)$, and $w$, the Secondary Variable $(S V)$, and has two outedges labeled $P V \neq S V$ and $P V=S V$. Each internal node represents the biconditional expansion with respect to $v, w$ :

$f(v, w, . ., z)=(v \oplus w) \cdot f\left(w^{\prime}, w, . ., z\right)+(v \bar{\oplus} w) \cdot f(w, w, . ., z)$ where the symbol $\oplus$ represents the XOR operator and $w^{\prime}$ is the complement of $w$.

The $P V \neq S V$ and $P V=S V$ edges connect to $f\left(w^{\prime}, w, . ., z\right)$ and $f(w, w, . ., z)$ functions, respectively. For the sake of simplicity, we refer hereafter to $f\left(w^{\prime}, w, . ., z\right)$ and $f(w, w, . ., z)$ as to $f_{v \neq w}$ and $f_{v=w}$, respectively. Functions of a single variable $v$ cannot be directly decomposed by the biconditional expansion in Eq. 1. In such a condition, $v$ is assigned to the $P V$ and a fictitious variable $w=1$ is introduced and assigned to the $S V$, collapsing the biconditional expansion into a Shannon's expansion. With this boundary condition, any Boolean function can be fully decomposed and represented with a BBDD.

\section{B. BBDD Ordering}

Constraining the order of variable appearance in every root to sink path is one of the requirements to achieve canonicity in BBDDs. For this purpose, the Chain Variable Order (CVO), introduced in [14], imposes a variable order on all root to sink paths for $P V \mathrm{~s}$ and a rule for the adjacent $S V$ s. Given a Boolean function $f$ and an input variable order $\pi=\left(\pi_{0}, \pi_{1}, . ., \pi_{n-1}\right)$, the $\mathrm{CVO}$ assigns $P V \mathrm{~s}$ and $S V \mathrm{~s}$ by levels as:

$\left\{\begin{array}{l}P V_{i}=\pi_{i} \\ S V_{i}=\pi_{i+1}\end{array} \quad\right.$ with $i=0,1, . ., n-2 ;\left\{\begin{array}{l}P V_{n-1}=\pi_{n-1} \\ S V_{n-1}=1\end{array}\right.$

CVO Example: From $\pi=(a, b, c)$, the corresponding CVO is obtained following Eq. 2. The consecutive ordering by couples $\left(P V_{i}, S V_{i}\right)$ is thus $((a, b),(b, c),(c, 1))$.

Note that the size of an ordered BBDD depends on the particular variable order $\pi$ assigned through the CVO. Dedicated re-ordering techniques are presented in Section IV-A4 to handle this issue.

\section{BBDD Reduction}

In order to improve the representation efficiency, BBDDs can be reduced according to a set of rules [14]. A BBDD is said reduced when it respects the following rules:

R1) It contains no two nodes, root of isomorphic subgraphs.

R2) It contains no nodes with identical children.

R3) It contains no empty levels.

R4) Subgraphs representing single variable functions degenerates into a single node with $\mathrm{SV}=1$, i.e., a BDD node.

Note that rules R1-2 are the straightforward extension of BDD [3] reduction rules. Then, rules R3-4 derive from the enhanced expressive power of the biconditional expansion [14].

\section{Reduced and Ordered BBDD Canonicity}

Reduced and ordered BBDDs are canonical, i.e., unique for a given input variable order $\pi=\left(\pi_{0}, \pi_{1}, . ., \pi_{n-1}\right)$ and corresponding CVO. Canonicity is preserved if a complement attribute is enabled at $P V \neq S V$ edges and only the 1 sink node is permitted. Formal proofs about BBDD canonicity are given in [14]. Unless specified otherwise, we refer hereafter to BBDDs as to ordered and reduced BBDDs.

BBDDs improve the expressive power of binary decision diagrams while remaining a canonical representation form. In order to fully harness such opportunity in EDA, a BBDD package is presented in the following section. 


\section{BBDD MANipulation PACKAGE}

This section presents an efficient manipulation theory for BBDDs and its practical software implementation [19]. Experimental results for the developed BBDD package are given and compared to a state-of-art BDD package.

\section{A. Efficient Manipulation of BBDDs}

Nowadays, one fundamental reason to keep decision diagrams small is not just to successfully fit them into the memory, that in a modern server could store up to 1 billion nodes, but more to maximize their manipulation performance. Following this trend, we design the BBDD manipulation algorithms and data structures aiming to minimize the runtime while keeping under control the memory footprint. The key concepts unlocking such target are (i) unique table to store BBDD nodes in a strong canonical form, (ii) recursive formulation of Boolean operations in terms of biconditional expansions with relative computed table, (iii) memory management to speed up computation and (iv) chain variable re-ordering to minimize the BBDD size. We discuss in details each point hereafter.

1) Unique Table: BBDD nodes must be stored in an efficient form, allowing fast lookup and insertion. Thanks to canonicity, BBDD nodes are uniquely labeled by a tuple $\{$ CVO-level, $\neq$-child, $\neq$-attribute, $=$-child $\}$. A unique table maps each tuple $\{$ CVO-level, $\neq$-child, $\neq$-attribute, $=$-child $\}$ to its corresponding BBDD node via a hash-function. Hence, each BBDD node has a distinct entry in the unique table pointed by its hash-function, enabling a strong canonical form representation for BBDDs.

Exploiting the strong canonical form, equivalence test between two BBDD nodes corresponds to a simple pointer comparison. Thus, lookup and insertion operations in the unique table are efficient. Before a new node is added to the BBDD, a lookup checks if its corresponding tuple \{CVO-level, $\neq$-child, $\neq$-attribute, $=$-child $\}$ already exists in the unique table and, if so, its pointed node is returned. Otherwise, a new entry for the node is created in the unique table.

2) Boolean Operations between BBDDs: The capability to apply Boolean operations between two BBDDs is essential to represent and manipulate large functions of interest in EDA. Consequently, an efficient algorithm to compute $f \otimes g$, where $\otimes$ is any Boolean function of two operands and $\{f, g\}$ are two existing BBDDs, is mandatory in the manipulation package. A recursive formulation of $f \otimes g$, in terms of biconditional expansions, allows us to take advantage of the information stored in the existing BBDDs and hence reduce the computation complexity. Algorithm 1 shows the outline of the recursive implementation for $f \otimes g$. The input of the algorithm are the BBDDs for $\{f, g\}$ and the two-operand Boolean function $\otimes$ that has to be computed between them. If $f$ and $g$ are identical, or one of them is the sink 1 node, the operation $f \otimes g$ reaches a terminal condition. In this case, the result is retrieved from a pre-defined list of trivial operations and returned immediately (Alg.1 $\alpha$ ). When a terminal condition is not encountered, the presence of $\{f, g, \otimes\}$ is first checked in a computed table, where previously performed operations are stored in case of later use. In the case of positive outcome, the result is retrieved from the computed table and returned immediately (Alg.1 $\beta$ ). Otherwise, $f \otimes g$ has to be explicitly computed

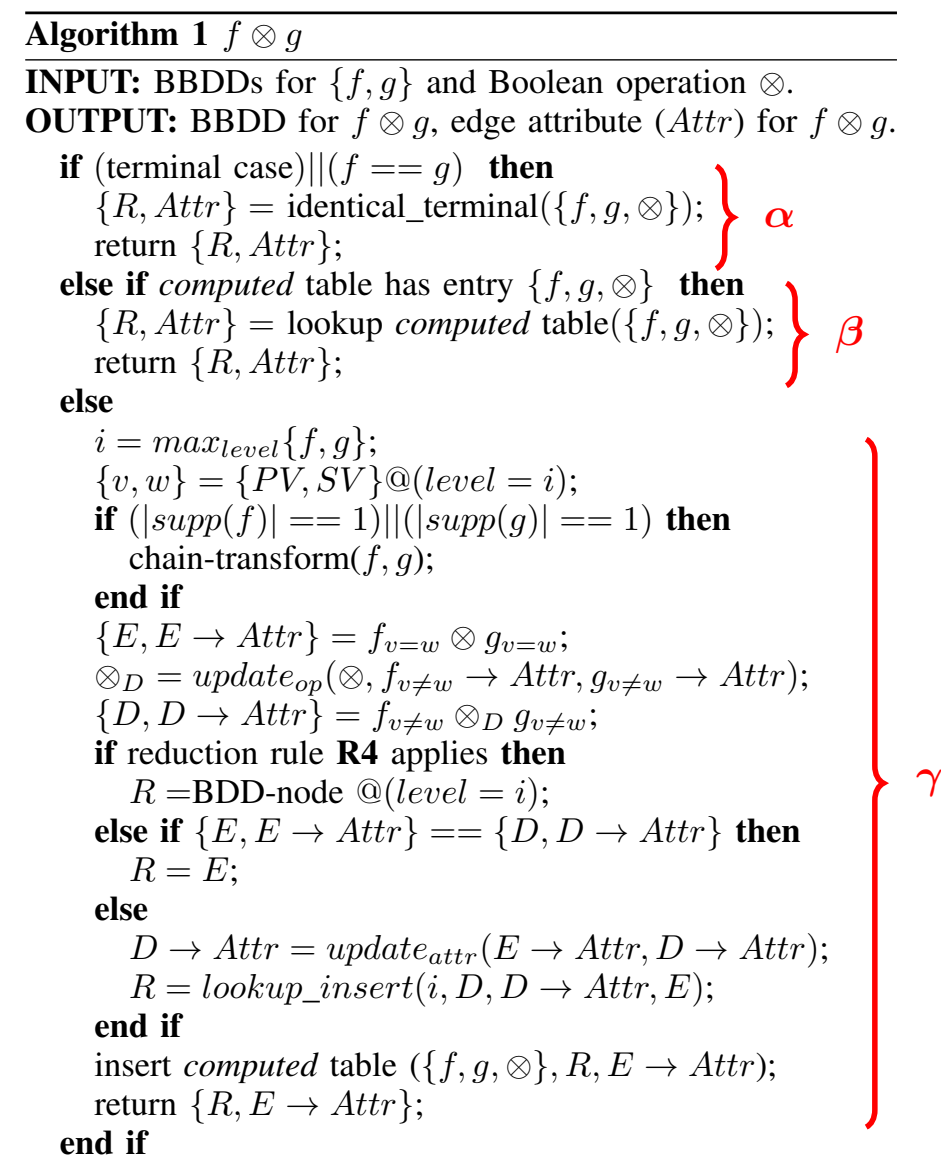

(Alg.1 $\gamma$ ). The top level in the CVO for $f \otimes g$ is determined as $i=\max _{\text {level }}\{f, g\}$ with its $\left\{P V_{i}=v, S V_{i}=w\right\}$. The root node for $f \otimes g$ is placed at such level $i$ and its children computed recursively. Before proceeding in this way, we need to ensure that the two-variable biconditional expansion is well defined for both $f$ and $g$, particularly if they are single variable functions. To address this case, single variable functions are prolonged down to $\min _{\text {level }}\{f, g\}$ through a chain of consecutive BBDD nodes. This temporarily, and locally, may violate reduction rule $\mathbf{R} 4$ to guarantee consistent $\neq-$ and $=-$ edges. However, rule $\mathbf{R 4}$ is enforced before the end of the algorithm. Provided such handling strategy, the following recursive formulation, in terms of biconditional expansions, is key to efficiently compute the children for $f \otimes g$ :

$$
f \otimes g=(v \oplus w)\left(f_{v \neq w} \otimes g_{v \neq w}\right)+(v \bar{\oplus} w)\left(f_{v=w} \otimes g_{v=w}\right)
$$

The term $\left(f_{v \neq w} \otimes g_{v \neq w}\right)$ represents the $\neq$-child for the root of $f \otimes g$ while the term $\left(f_{v=w} \otimes g_{v=w}\right)$ represents the $=$-child. In $\left(f_{v \neq w} \otimes g_{v \neq w}\right)$, the Boolean operation $\otimes$ needs to be updated according to the regular/complemented attributes appearing in the edges connecting to $f_{v \neq w}$ and $g_{v \neq w}$. After the recursive calls for $\left(f_{v=w} \otimes g_{v=w}\right)$ and $\left(f_{v \neq w} \otimes g_{v \neq w}\right)$ return their results, reduction rule $\mathbf{R} \mathbf{4}$ is applied. Finally, the tuple $\{$ top-level, $\neq$ child, $\neq$-attribute, $=$-child $\}$ is found or added in the unique table and its result updated in the computed table.

Observe that the maximum number of recursions in Eq. 3 is determined by all possible combination of nodes between 

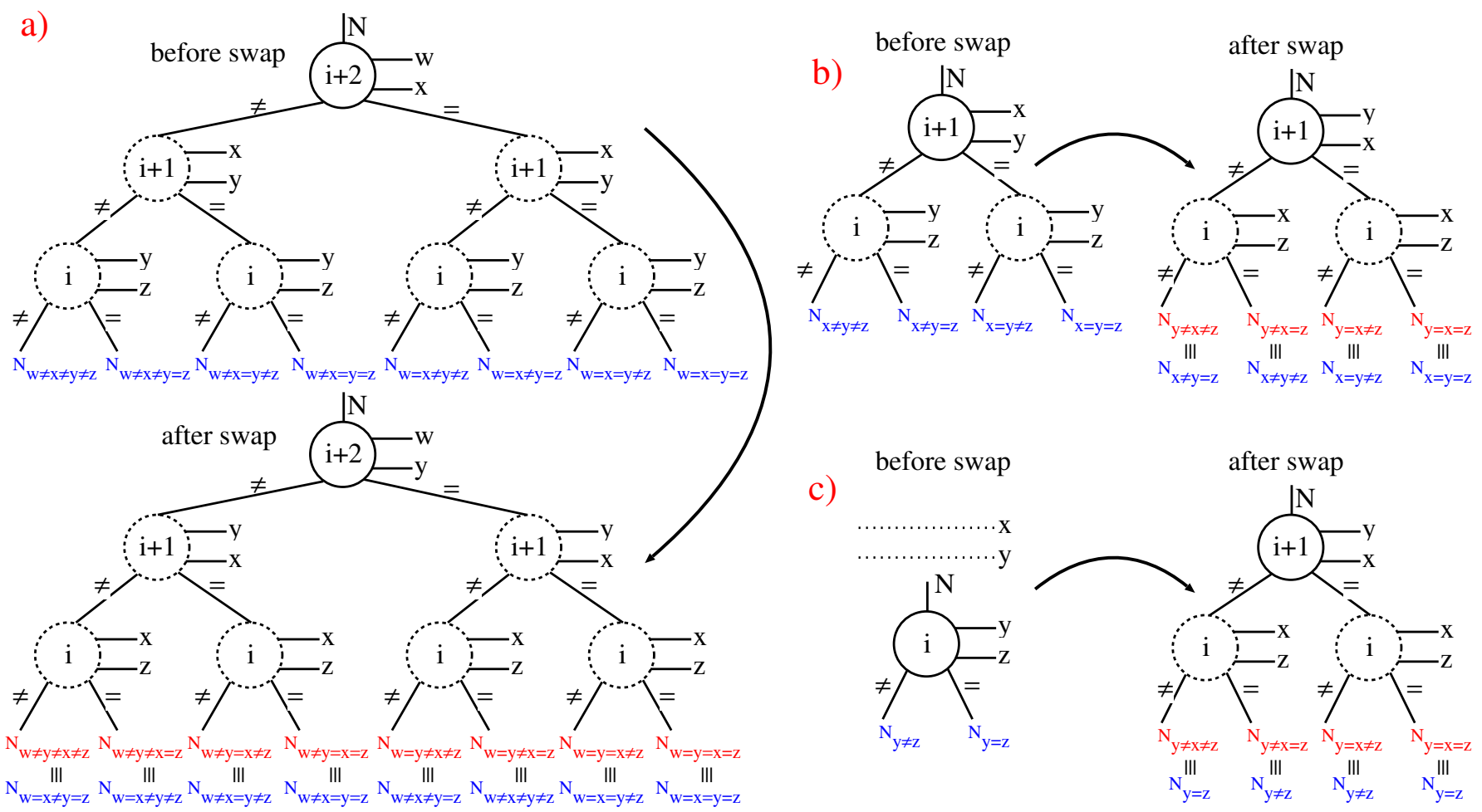

Fig. 2: Variable swap $i \rightleftharpoons i+1$ involving the CVO levels $\left(P V_{i+2}=w, S V_{i+2}=x\right),\left(P V_{i+1}=x, S V_{i+1}=y\right)$ and $\left(P V_{i}=y\right.$, $S V_{i}=z$ ). Effect on nodes at level $i+2$ (a) $i+1$ (b) and $i$ (c).

the BBDDs for $f$ and $g$. Assuming constant time lookup in the unique and computed tables, it follows that the time complexity for Algorithm 1 is $O(|f| \cdot|g|)$, where $|f|$ and $|g|$ are the number of nodes of the BBDDs of $f$ and $g$, respectively.

3) Memory Management: The software implementation of data-structures for unique and computed tables is essential to control the memory footprint but also to speed-up computation. In traditional logic manipulation packages [20], the unique and computed tables are implemented by a hash-table and a cache, respectively. We follow this approach in the BBDD package, but we add some specific additional technique to further speed-up computation. Informally, we minimize the access time to stored nodes and operations by dynamically changing the data-structure size and hashing function, on the basis of a $\{$ size $\times$ access-time $\}$ quality metric.

The core hashing-function for all BBDD tables is the Cantor pairing function between two integer numbers [21]:

$$
C(i, j)=0.5 \cdot(i+j) \cdot(i+j+1)+i
$$

which is a bijection from $\mathbb{N}_{0} \times \mathbb{N}_{0}$ to $\mathbb{N}_{0}$ and hence a perfect hashing function [21]. In order to fit the memory capacity of computers, modulo operations are applied after the Cantor pairing function allowing collisions to occur. To limit the frequency of collisions, a first modulo operation is performed with a large prime number $m$, e.g., $m=15485863$, for statistical reasons. Then, a final modulo operation resizes the result to the current size of the table.

Hashing functions for unique and computed tables are obtaining by nested Cantor pairings between the tuple elements with successive modulo operations.
Collisions are handled in the unique table by a linked list for each hash-value, while, in the computed table, the cache-like approach overwrites an entry when collision occurs.

Keeping low the frequency of collisions in the unique and computed table is of paramount importance to the BBDD package performance. Traditional garbage collection and dynamic table resizing [20] are used to address this task. When the benefit deriving by these techniques is limited or not satisfactory, the hash-function is automatically modified to rearrange the elements in the table. Standard modifications of the hash-function consist of nested Cantor pairings re-ordering and re-sizing of the prime number $m$.

4) Chain Variable Re-ordering: The chain variable order for a BBDD influences the representation size and therefore its manipulation complexity. Automated chain variable reordering assists the BBDD package to boost the performance and reduce the memory requirements. Among traditional variable re-ordering techniques for binary decision diagrams, we extend Rudell's sifting algorithm [9]. Thanks to its general formulation, the sifting algorithm [9] can be directly extended to BBDDs, but a new swap theory handling the chain variable order is needed.

Variable swap in the CVO exchanges the $P V \mathrm{~s}$ of two adjacent levels $i$ and $i+1$ and updates the neighbor $S V \mathrm{~s}$ accordingly. The effect on the original variable order $\pi$, from which the CVO is derived as per Eq. 2, is a direct swap of variables $\pi_{i}$ and $\pi_{i+1}$. Note that all the nodes/functions concerned during a CVO swap are overwritten (hence maintaining the same pointer) with the new tuple generated at the end of the operation. In this way, the effect of the CVO swap remains local, as the edges of the above portion of the BBDD still point 
TABLE I: Experimental Results for the BBDD Manipulation Package

\begin{tabular}{|c|c|c|c|c|c|c|c|c|}
\hline Benchmarks & Inputs & Outputs & \multicolumn{2}{|c|}{ BBDD Package (this work) } & \multicolumn{3}{c|}{ BDD Package (CUDD) } \\
\hline \multicolumn{2}{|c|}{} & & Node Count & Build time (s) & Sift time (s) & Node Count & Build time (s) & Sift time (s) \\
\hline C1355 & 41 & 32 & 54225 & 0.23 & 0.11 & 74056 & 0.06 & 0.59 \\
\hline C1908 & 33 & 25 & 14918 & 0.06 & 0.23 & 17980 & 0.09 & 0.34 \\
\hline C499 & 41 & 32 & 135784 & 1.56 & 3.21 & 160691 & 3.04 & 4.28 \\
\hline seq & 41 & 35 & 4554 & 0.07 & 0.33 & 5607 & 0.14 & 0.44 \\
\hline my_adder & 33 & 17 & 166 & 0.13 & 0.15 & 1006 & 0.15 & 0.14 \\
\hline frg1 & 28 & 3 & 284 & $<0.01$ & $<0.01$ & 296 & $<0.01$ & $<0.01$ \\
\hline misex3 & 14 & 14 & 745 & 0.02 & $<0.01$ & 885 & 0.03 & 0.02 \\
\hline misex1 & 8 & 7 & 51 & $<0.01$ & $<0.01$ & 68 & $<0.01$ & $<0.01$ \\
\hline comp & 32 & 3 & 97 & $<0.01$ & $<0.01$ & 330 & 0.23 & 0.67 \\
\hline count & 35 & 16 & 328 & $<0.01$ & $<0.01$ & 342 & $<0.01$ & 0.01 \\
\hline cordic & 23 & 2 & 54 & $<0.01$ & $<0.01$ & 80 & $<0.01$ & 0.01 \\
\hline alu4 & 14 & 8 & 1076 & $<0.01$ & $<0.01$ & 897 & $<0.01$ & $<0.01$ \\
\hline C17 & 5 & 2 & 15 & $<0.01$ & $<0.01$ & 13 & $<0.01$ & $<0.01$ \\
\hline 9symml & 9 & 1 & 19 & $<0.01$ & $<0.01$ & 25 & $<0.01$ & $<0.01$ \\
\hline z4ml & 7 & 4 & 21 & $<0.01$ & $<0.01$ & 37 & $<0.01$ & $<0.01$ \\
\hline decod & 5 & 16 & 46 & $<0.01$ & $<0.01$ & 96 & $<0.01$ & $<0.01$ \\
\hline parity & 16 & 1 & 9 & $<0.01$ & $<0.01$ & 17 & $<0.01$ & $<0.01$ \\
\hline \hline Average & 22.64 & 12.82 & $1.24 \mathrm{e} 04$ & 0.12 & 0.24 & $1.54 \mathrm{e} 04$ & 0.22 & 0.37 \\
\hline
\end{tabular}

to the same logical function.

A variable swap $i \rightleftharpoons i+1$ involves three CVO levels $\left(P V_{i+2}=w, S V_{i+2}=x\right),\left(P V_{i+1}=x, S V_{i+1}=y\right)$ and $\left(P V_{i}=y, S V_{i}=z\right)$. The level $i+2$ must be considered as it contains in $S V$ the variable $x$, which is the $P V$ swapped at level $i+1$. If no level $i+2$ exists $(i+1$ is the top level) the related operations are simply skipped. In the most general case, each node at level $i+2, i+1$ and $i$ has 8,4 and 2 possible children on the portion of BBDD below level $i$. Some of them may be identical, following to reduction rules R1-4, or complemented, deriving by the $\neq$-edges attributes in their path. Fig. 2 depicts the different cases for a general node $N$ located at level $i+2, i+1$ or $i$, with all their possible children. After the swap $i \rightleftharpoons i+1$, the order of comparisons $w \star x \star y \star z$ is changed to $w \star y \star x \star z$ and the children of $N$ must be rearranged consequently $(\star \in\{=, \neq\}$ ). Using the transitive property of equality and congruence in the binary domain, it is possible to remap $w \star x \star y \star z$ into $w \star y \star x \star z$ as:

$$
\begin{aligned}
& \star \in\{=, \neq\}, \quad \star:\{=, \neq\} \rightarrow\{\neq,=\} \\
& \left(w \star_{i+2} x=y \star_{i} z\right) \rightarrow\left(w \star_{i+2} y=x \star_{i} z\right) \\
& \left(w \star_{i+2} x \neq y \star_{i} z\right) \rightarrow\left(w \star_{i+2} y \neq x \star_{i} z\right)
\end{aligned}
$$

Following remapping rules in Eq. 5, the children for $N$ can be repositioned coherently with the variable swap. In Fig.2, the actual children rearrangement after variable swap is shown. In a bottom-up approach, it is possible to assemble back the swapped levels, while intrinsically respecting reduction rules R1-4, thanks to the unique table strong canonical form.

Based on this CVO swap theory, the BBDD re-ordering (sifting) algorithm is derived from [9] and works as follows. Let $n$ be the number of variables in the initial order $\pi$. Each variable $\pi_{i}$ is considered in succession and the influence of the other variables is locally neglected. Swap operations are performed to move $\pi_{i}$ in all $n$ potential positions in the CVO. The best BBDD size encountered is remembered and its $\pi_{i}$ position in the $\mathrm{CVO}$ is restored at the end of the variable processing. This procedure is repeated for all variables. It follows that BBDD sifting requires $O\left(n^{2}\right)$ swap operations.

\section{B. BBDD Results}

We have developed a BBDD package in $\mathrm{C}$ language implementing the techniques described in this work. The package is available online at [19] and consists of about 4k lines of code. For the sake of comparison, we considered the latest release of CUDD [20], the state-of-art manipulation package for BDDs. BDDs [20] and BBDDs [19] packages run on a Xeon X5650 24-GB RAM machine. The benchmarks considered are taken from the traditional MCNC suite.

CUDD [20] receives as input a BLIF format description of a combinational logic network. BDDs are first built using the initial order provided in the BLIF file and later sifted [9].

The BBDD package [19] receives as input a Verilog description of a combinational logic network, flattened onto primitive Boolean operations (XOR, AND, OR, INV, BUF). Then, it provides as output a Verilog description for the built BBDD together with its log information. As for the BDDbased counterpart, BBDDs are first built using the initial order provided in the Verilog file and later sifted.

Table I shows experimental results for the BBDD package [19] and CUDD package [20]. On average, BBDDs built and sifted by the package developed in this work are $19.48 \%$ smaller, in terms of node count, with respect to BDDs built and sifted by CUDD [20]. This is thanks to the expressive power of BBDDs, where the branching decision at each node is biconditional on two variables per time, rather than only one as in standard BDDs. Moreover, the building and sifting time are faster for BBDDs than for BDDs, achieving an overall speed-up factor of $1.63 \mathrm{x}$ as compared to CUDD [20]. The reason for such speed-up is twofold. On the one hand, BBDDs have fewer nodes than BDDs so the number of operations required is directly reduced. On the other hand, the BBDDpackage [19] has a memory management strategy expressly targeting low collision frequency, further boosting the package performance.

\section{Case Study: Datapath Synthesis Front-End}

This section showcases the interest of the BBDD package in the automated design of datapath circuits. A BBDD-based 
TABLE II: Experimental Results for the BBDD-based Datapath Synthesis

\begin{tabular}{|c|c|c|c|c|c|c|c|c|}
\hline Benchmarks & Inputs & Outputs & \multicolumn{3}{|c|}{ BBDD Package + Commercial Synthesis Flow } & \multicolumn{3}{|c|}{ Commercial Synthesis Flow } \\
\hline & & & Area $\left(\mu m^{2}\right)$ & Delay $(n s)$ & Gate Count & Area $\left(\mu m^{2}\right)$ & Delay $(n s)$ & Gate Count \\
\hline Adder 32 & 64 & 33 & 41.01 & 2.17 & 186 & 45.98 & 3.42 & 216 \\
\hline Adder 64 & 128 & 65 & 83.05 & 4.46 & 380 & 93.02 & 7.01 & 440 \\
\hline Equality 32 & 64 & 1 & 17.78 & 0.11 & 63 & 18.27 & 0.18 & 72 \\
\hline Equality 64 & 128 & 1 & 35.57 & 0.13 & 119 & 36.18 & 0.20 & 136 \\
\hline Magnitude 32 & 64 & 1 & 13.65 & 0.82 & 41 & 21.77 & 1.16 & 186 \\
\hline Magnitude 64 & 128 & 1 & 29.44 & 1.64 & 102 & 44.17 & 2.30 & 378 \\
\hline Barrel 32 & 39 & 32 & 71.68 & 0.50 & 545 & 76.44 & 0.50 & 569 \\
\hline Barrel 64 & 70 & 64 & 165.42 & 0.58 & 1255 & 178.50 & 0.60 & 1320 \\
\hline Average & 85.62 & 24.75 & 57.20 & 1.30 & 336.38 & 64.29 & 1.92 & 414.62 \\
\hline
\end{tabular}

synthesis methodology is proposed and compared to a commercial synthesis flow, for a $22 \mathrm{~nm}$ CMOS technology.

\section{A. Re-writing Datapaths with BBDDs}

Datapath circuits are essential components in today's integrated circuits. Standard synthesis techniques face challenges to optimize datapaths, as they are dominated by arithmetic operations which are not natively supported by conventional logic representation forms. Differently, BBDD nodes inherently act as two-variable comparators, a basis function for arithmetic operations. This feature enables datapaths to be efficiently represented with BBDDs. Nevertheless, the BBDD representation efficiency is not just limited to datapaths. Motivated by this consideration, we employ the BBDD package as front-end to a commercial synthesis tool to pre-structure datapaths and facilitate their synthesis.

\section{B. Synthesis Results}

Adder, comparator and shifter datapaths are considered, in operand 32 and 64 bit-widths, as synthesis benchmarks. They are written in Verilog language. A standard cell library consisting of MAJ-3, XOR-2, XNOR-2, NAND-2, NOR-2 and INV logic gates is characterized in CMOS $22 \mathrm{~nm}$ technology [22] and used to support the synthesis flow. When employed stand-alone, the commercial synthesis tool identifies arithmetic building blocks in datapaths and optimizes their corresponding implementation. We use the BBDD package to rewrite datapaths prior to the synthesis with the commercial tool. In this approach, such synthesis tool may be precluded to identify arithmetic operations but forced to ordinarily synthesize the restructured circuit, making the BBDD representation the main responsible for the quality of the designed datapath.

Table II shows synthesis results. On average, datapath designed by BBDD re-writing followed by the standard synthesis tool are $11.02 \%$ smaller and $32.29 \%$ faster as compared to the same circuits designed by the standard synthesis tool alone. BBDD representation is capable to evidence advantageous logic structures in datapath, not apparent with traditional synthesis techniques.

\section{CONCLUSIONS}

We presented the theory, and software implementation, for efficient manipulation of Biconditional Binary Decision Diagrams (BBDDs), a novel class of decision diagrams based on an equality/inequality switching paradigm. As compared to a state-of-art decision diagram manipulation package, the developed BBDD software achieves an average node count reduction of $19.48 \%$ and a speed-up factor of $1.63 \mathrm{x}$, measured over a standard set of logic benchmarks. The key factors enabling such improvements are (i) strong canonical form preconditioning of stored BBDD nodes, (ii) recursive formulation of Boolean operations in terms of biconditional expansions, (iii) performance-oriented memory management and (iv) dedicated BBDD re-ordering techniques. Employed in the synthesis of datapaths, the BBDD manipulation package is capable to advantageously restructure arithmetic operations producing $11.02 \%$ smaller and $32.29 \%$ faster circuits, as compared to a commercial synthesis flow.

\section{ACKNOWLEDGEMENTS}

This research was supported by ERC-2009-AdG-246810.

\section{REFERENCES}

[1] C.Y. Lee, Representation of Switching Circuits by Binary-Decision Programs, Bell Systems Technical Journal, 1959.

[2] S.B. Akers, Binary Decision Diagrams, IEEE Trans. Comp., C-27(6):509516, June 1978.

[3] R.E. Bryant, Graph-based algorithms for Boolean function manipulation, IEEE Transactions on Computers, C-35: 677-691, 1986.

[4] C. Yang and M. Ciesielski, BDS: A BDD-Based Logic Optimization System, IEEE Trans. CAD, vol. 21, pp. 866-876, July 2002.

[5] S. Malik et al., Logic verification using binary decision diagrams in a logic synthesis environment, Proc. ICCAD, 1988.

[6] M.S. Abadir et al., Functional test generation for digital circuits using binary decision diagrams, IEEE Trans. Comput., C35 (1986), pp.375-379.

[7] C. Scholl, R. Drechsler, B. Becker, Functional simulation using binary decision diagrams, Proc. ICCAD, 1997.

[8] K.S. Brace, R.L. Rudell, R.E. Bryant, Efficient implementation of a BDD package, Proc. DAC, 1990.

[9] R. Rudell, Dynamic variable ordering for ordered binary decision diagrams, Proc. ICCAD, 1993.

[10] R.E. Bryant, On the Complexity of VLSI Implementations and Graph Representations of Boolean Functions with Application to Integer Multiplication, IEEE Trans. on Comp., vol. 40, no. 2, p. 205, February 1991.

[11] S. Minato, Zero-suppressed BDDs for set manipulation in combinatorial problems, Proc. DAC, pp. 272-277, 1993

[12] R. Drechsler et al., Ordered Kronecker functional decision diagrams: a data structure for representation and manipulation of Boolean functions, IEEE Trans. CAD, Vol. 17, Issue 10, pp. 965-973, Oct. 1998.

[13] E.I. Goldberg, Y. Kukimoto, R.K. Brayton, Canonical TBDD's and Their Application to Combinational Verification, Proc. IWLS, 1997.

[14] L. Amaru, P.-E. Gaillardon, G. De Micheli, Biconditional BDD: A Novel Canonical Representation Form Targeting the Synthesis of XORrich Circuits, Proc. DATE 2013.

[15] R. Drechsler, B. Becker, Binary Decision Diagrams: Theory and Implementation, Kluwer Academic Publisher, 1998.

[16] M. De Marchi et al., Polarity control in Double-Gate, Gate-All-Around Vertically Stacked Silicon Nanowire FETs, Proc. IEDM 2012.

[17] Y. Lin et al., High-Performance Carbon Nanotube Field-Effect Transistor with Tunable Polarities, IEEE Trans. Nanotech., 4(5): 481-489, 2005.

[18] D. Lee et al., Combinational Logic Design Using Six-Terminal NEM Relays, IEEE Trans. CAD, Vol. 32, Issue: 5, pp-653-666, May 2013.

[19] BBDD package available at: http://lsi.epfl.ch/page-102566-en.html.

[20] CUDD: CU Decision Diagram Package Release 2.5.0

[21] P. Tarau, Pairing Functions, Boolean Evaluation and Binary Decision Diagrams, Proc. CICLOPS, 2008

[22] Predictive Technology Model (PTM), http://ptm.asu.edu/ 